\title{
La Sodeci, Un Outil Strategique Dans La Distribution D’eau Potable En Cote d'Ivoire
}

\author{
Atta Koffi \\ Maître de Recherches Cames, Université Félix Houphouët Boigny, \\ Institut de Géographie Tropicale, LARES \\ Diabagate Abou \\ Enseignant-Chercheur, Université Félix Houphouët Boigny, \\ Institut de Géographie Tropicale, LARES \\ Konan Honorée Ghislaine \\ Doctorante, Université Félix Houphouët Boigny, \\ Institut de Géographie Tropicale, LARES
}

doi: 10.19044/esj.2016.v12n20p153 URL:http://dx.doi.org/10.19044/esj.2016.v12n20p153

\begin{abstract}
This study aims to make SODECI (water distribution) better known by the Ivorian populations. Indeed, very few studies have been made on it to make it known. Therefore, this study raises the problem of ignorance of this strategic tool in the supply of drinking water in Côte d'Ivoire. To solve this problem, a methodology based on documentary research and interview has allowed to track its development route. Thus, from its creation, it will undergo a remarkable development but it will later face crises that will affect it without undermining its fundamentals. Until late 2015, it served 964 villages and 13,745,067 inhabitants that is a national coverage of $68 \%$. The fruit of his prowess is put to the credit of an exceptional managerial policy and a civic attitude during the Ivorian crisis that rocked and split the country in 2002.
\end{abstract}

Keywords: Ivory Coast, Sodeci, drinking water, priority, strategy

\section{Résumé}

La présente étude vise à mieux faire connaître la Société de Distribution d'Eau de la Côte d'Ivoire (SODECI) aux populations ivoiriennes. En effet, rare sont les études réalisées sur elle pour la faire connaître, malgré son monopole sur toute l'étendue du territoire national. De ce fait, l'étude pose le problème de la méconnaissance de cet outil stratégique dans l'approvisionnement en eau potable en Côte d'Ivoire. Pour résoudre ce problème, une méthodologie basée sur la recherche 
documentaire et l'entretien a permis de relater son itinéraire de développement. Ainsi, à sa création, elle va connaître un développement remarquable mais elle sera confrontée plus tard à des crises qui vont l'affecter sans toutefois ébranler ses fondamentaux. Jusqu'en fin 2015, elle desservait 964 localités et 13745067 habitants soit un taux de couverture nationale de 68\%. Le fruit de ses prouesses est à mettre à l'actif d'une politique managériale d'exception et une attitude citoyenne face à la crise ivoirienne qui a secoué et divisé le pays en 2002.

Mots clés : Côte d’Ivoire, SODECI, eau potable, priorité, stratégie

\section{Introduction}

L'accès à l'eau potable est un enjeu capital pour l'ensemble de l'humanité,aussi les instances internationales l'ont-elles inscrit dans les Objectifs du Millénaire pour le Développement (OMD). La pertinence de cet enjeu pour l'ONU (2015) réside dans le fait que la proportion de personnes ayant accès de façon durable à l'eau de boisson potable reste faible face à une demande croissante. En réalité, il y a 884 millions de personnes dans le monde qui n'ont pas accès à une eau potable de qualité. Environ deux millions de personnes, pour la plupart des jeunes enfants, meurent chaque année des suites de maladies causées par une eau impropre à la consommation (ONU, 2015).

La Côte d'Ivoire, qui a très tôt compris cette exigence, a développé dès son indépendance une stratégie d'alimentation en eau potable par la création de la SODECI. Celle-ci a enregistré des résultats satisfaisants au fil des ans. Cette politique, ponctuée par des réformes innovantes, a permis selon les contextes, de structurer les besoins en trois sous-secteurs : l'hydraulique urbaine (HU), l'hydraulique villageoise (HV) et l'hydraulique villageoise améliorée (HVA). Pour Koukougnon (2012), l’amélioration des conditions de vie des populations ivoirienne est du ressort de l'Etat. Elle passe par un meilleur accès aux services de base dont l'eau potable. Cette volonté régalienne s'est traduite en action par la mise en œuvre du Programme National de l'Hydraulique Humaine (PNHH) dès 1973.

En effet, le levier principal sur lequel l'Etat de Côte d'Ivoire s'est appuyé pour réaliser la politique d'approvisionnement en eau potable est la SODECI (Société de Distribution d'Eau de la Côte-d’Ivoire). Chargée de la commercialisation et de la distribution de l'eau potable en Côte d'Ivoire, la SODECI a aujourd'hui plus de cinquante ans d'expérience et près de dix millions d'abonnés. C'est le plus ancien et le plus important partenariat public privé depuis les débuts de l'indépendance (SODECI, 2010). Solidement implantée en Côte d'Ivoire, elle a enregistré des progrès remarquables dans l'accès à l'eau potable tout au long de ces années et a fait 
de la qualité et de la distribution de l'eau ses priorités. Même si la crise politico-militaire de 2002 a fortement touché le secteur de l'eau, ses fondamentaux demeurent toujours solides.

Cependant, cet outil indispensable ne semble pas être très bien connu de nombreux ivoiriens. Il ne l'est que pour le règlement d'abonnements et de litiges ou pour le paiement des factures. De ce fait, le problème que pose cette étude est la méconnaissance de la SODECI par les populations ivoiriennes. L’objectif assigné à cette réflexion est donc de faire connaître cette structure aux Ivoiriens en élucidant son itinéraire de développement. Aussi notre étude présentera- t-elle d'une part les priorités de la SODECI et d'autre part, les difficultés qu’elle a vécu. Mais quelle est la méthode appropriée pour cerner cette problématique?

\section{Methodologie}

Pour la collecte de nos informations, nous avons eu recours à deux méthodes spécifiques qui sont la recherche documentaire et l'entretien. La recherche documentaire a consisté à recueillir, à travers les ouvrages, toutes les informations concernant la SODECI afin de la circonscrire. Quant aux entretiens, ils se sont déroulés d'une part, auprès des responsables de la SODECI pour avoir des informations et des données sur l'itinéraire de développement de l'entreprise depuis sa création, et d'autre part, auprès des populations abonnées afin de recueillir leur opinion sur la SODECI.

\section{1-Les priorites de developpement de la sodeci}

Ce volet s'articule autour de l'historique de la naissance de la Sodeci et des priorités qu'elle s’est accordée.

\section{1-1-l'historique de la naissance de la SODECI}

La SODECI est née à la suite d'un appel d'offre. En effet, à la fin de l'année 1959, la mairie d'Abidjan a lancé un appel d'offre pour un contrat de concession pour l'exploitation des services d'eau à Abidjan et sur le territoire ivoirien et c'est la Société d'Aménagement Urbain et Rural (SAUR) qui a obtenu le marché. C'est ainsi que la Société de Distribution d'Eau de Côte d'Ivoire voit le jour avec un capital de 40 millions de franc CFA entièrement détenu par la SAUR. La SODECI se substitue dès le 27 septembre 1960 à la maison mère, dans tous ses droits et ses obligations. A l'époque, les services d'eau dans le pays étaient placés sous la responsabilité municipale. La SODECI va progressivement étendre son réseau à d'autres villes. A partir de 1973, le Programme National de l'Hydraulique Humaine (PNHH) va permettre à la SODECI de prendre définitivement son envol et de se développer pour devenir une société de service public de référence en Afrique. Aujourd’hui, la SODECI est une société privée dotée d’un capital 
de 4,5 milliards de francs CFA et dessert 964 localités et 13745067 abonnés soit un taux de couverture de 68\% sur le plan national (ONEP, 2015). Son savoir-faire s'exporte dans toute l'Afrique de l'Ouest. Le fruit de ses prouesses est à mettre à l'actif d'une politique managériale d'exception innovée par son ex-président et une attitude citoyenne face à la crise ivoirienne qui a secoué et divisé le pays en 2002. La SODECI est en effet, l'une des rares entreprises à avoir poursuivi le service malgré les difficultés dans les ex-zones Centre Nord Ouest (SODECI, 2010).

\section{1-2-Les priorités de la Sodeci}

A sa création, la SODECI s’est fixée des priorités. Il s’agissait pour elle de maîtriser la qualité de l'eau (1960) ; de faire face à l'urbanisation croissante de la Côte d'Ivoire (1960-1972); de se développer sur le plan national (1973-1987) ; d'étendre le réseau à toute la population (1987-2007) et d’améliorer et préserver le patrimoine (2008-2022) (SODECI, 2010).

\section{1-2-1-Maîtriser la qualité de l'eau (1960)}

La SODECI démarre timidement ses activités avec un champ d'action restreint et des moyens limités. Elle rencontre des difficultés organisationnelles tant au niveau technique qu'au niveau commercial. En effet, la situation gérée jusque-là par l'ancienne régie municipale est très précaire. Certains quartiers ne peuvent être alimentés que pendant quelques heures par jour. La vétusté de certaines installations affecte la qualité de l'eau distribuée. Chargée en fer et trop saline, l'eau a un goût désagréable. On constate également à l'époque de nombreuses fuites sur le réseau et l'absence de compteurs chez un grand nombre d'abonnés. C’est alors qu'intervient le programme de redressement de 1960 (SODECI, 2010).

Ce redressement est mené avec la réalisation de forages et la pose de canalisations, afin de desservir le sud d'Abidjan. Dès juin 1960, l'ensemble de la ville est alimenté en eau sans la moindre interruption. Pour améliorer le rendement du réseau, des plans détaillés de l'ensemble des canalisations sont élaborés et les branchements sont systématiquement relevés. Ces mesures permettent l'installation de 1600 compteurs chez les abonnés non équipés ou clandestins et de raccorder un millier de nouveaux abonnés. Le rendement de la nouvelle société passe ainsi de 55\% à 75\% en 15 mois, objectif fixé lors de la signature du contrat. Après avoir résolu les problèmes hérités du passé, la SODECI va commencer à relever le défi de l'extension de la ville d'Abidjan et de sa démographie galopante.

\section{1-2-2-Faire face à l'urbanisation croissante (1960-1972)}

Sur cette période, vu la dynamique des activités économiques et les progrès que connait l'économie ivoirienne, on assiste à un afflux massif des 
étrangers vers la Côte d'Ivoire en général et vers Abidjan en particulier (environ 30\% en 1972). Ainsi, la population de la ville d'Abidjan va plus que tripler, passant de 150000 à 636000 habitants au début des années 1970 (Diabagaté, 2012). Ce boom démographique va multiplier par 7,6 le nombre d'abonnés, par 2,7 la production en eau et par 3,7 le linéaire du réseau en douze ans, comme l'indique le tableau 1. La SODECI, qui couvrait essentiellement la ville d'Abidjan, va devoir répondre à une demande beaucoup plus forte en eau potable.

Tableau 1 : Données de la Sodeci à Abidjan entre 1960 et 1972

\begin{tabular}{|c|c|c|}
\hline Années & $\mathbf{1 9 6 0}$ & $\mathbf{1 9 7 2}$ \\
\hline Nombre d'abonnés & 3947 & 29902 \\
\hline Production $\left(\mathrm{km}^{3}\right)$ & 11400 & 31132 \\
\hline Facturation $\left(\mathrm{km}^{3}\right)$ & 6300 & 27338 \\
\hline Linéaire du réseau à Abidjan $(\mathrm{km})$ & 176 & 646 \\
\hline
\end{tabular}

Source : SODECI, 2010

\section{1-2-3-Se développer sur le plan national (1973-1987)}

En 1974, sur le plan national, seulement 34\% des ménages avaient accès à l'eau et de nombreuses villes n’étaient pas équipées d'un réseau de distribution. Le Gouvernement ivoirien a décidé de lancer un Programme National de l'Hydraulique (PNH) et de confier tous les équipements d'alimentation en eau au Ministère du Plan et le financement des programmes au Ministère de l'Economie et des Finances. Ce programme avait pour objectifs principaux d'équiper en adduction d'eau toutes les préfectures et sous-préfectures non encore pourvues avant la fin de l'année 1980 et de créer 7160 points d'eau pour les villages de plus de 100 habitants entre 1973 et 1980.

Un Fonds National de l'Hydraulique est également créé en vue de soutenir les gros investissements publics du secteur. Il a pour mission de contracter les emprunts, recueillir la part des recettes de ventes collectées par la SODECI revenant à l'Etat et d'assurer le service de la dette pour ces emprunts. Au moment où d'autres pays d'Afrique de l'Ouest nationalisent leurs sociétés d'eau, la Côte d'Ivoire renforce son partenariat public/privé par un contrat d'affermage sur l'ensemble du territoire national confiant ainsi à la SODECI, la responsabilité de l'exploitation de tous les ouvrages hydrauliques du pays. Le Programme National de l'Hydraulique entre alors dans sa phase active en 1974 et sera presque entièrement réalisé en 1980 avec l'établissement de nouvelles exploitations sur tout le territoire. La structure organisationnelle de la SODECI va également évoluer. Les services centraux vont se restructurer avec la création d'une direction de l'exploitation s'occupant des centres de l'intérieur et d'une direction technique coiffant la production, la qualité (contrôle) et la réalisation. Durant cette période, des directions régionales sont ouvertes à Korhogo, Yamoussoukro, Abengourou, 
Gagnoa, Abidjan et Man (SODECI, 2010). A ce jour, la SODECI compte douze (12) directions régionales regroupant environ cent soixante quinze (175) agences sur tout l'ensemble du territoire national (Cf. figure 1).

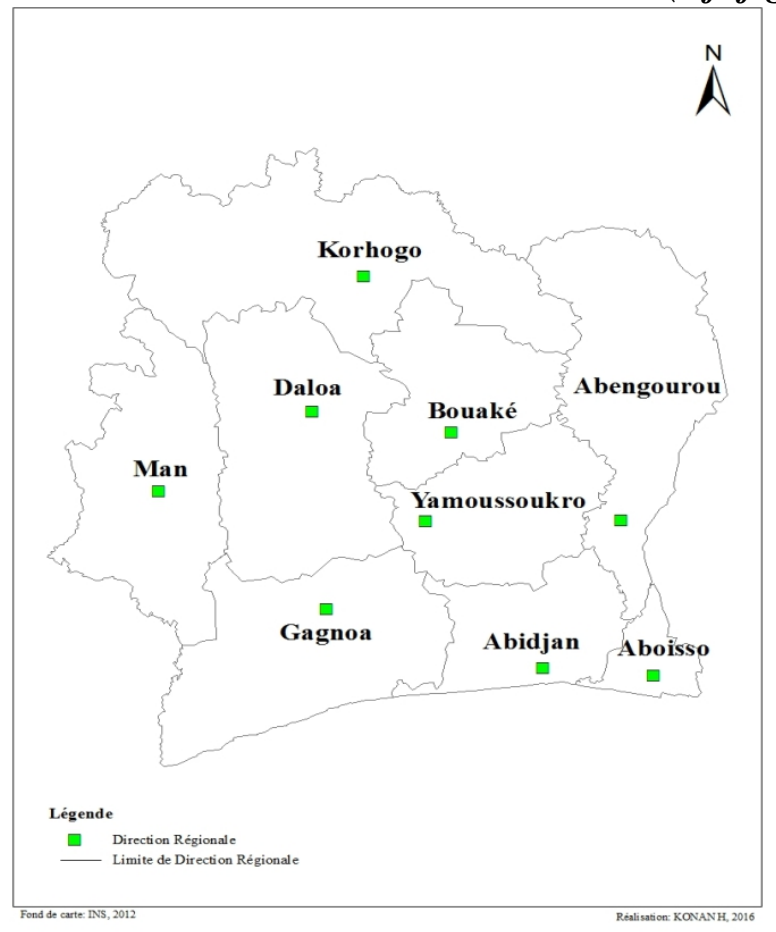

Figure 1 : Localisation des villes abritant les directions de la Sodeci en Côte d'Ivoire

\section{1-2-4-Etendre le réseau à toute la population (1987-2007)}

Cette période a vu le nombre d'abonnés de la SODECI s'accroître, passant de 212624 abonnés en 1988 à 576552 en 2007 (Cf. figure 2). Ce nombre a pratiquement triplé en dix neuf ans comme l’indique le tableau 2.

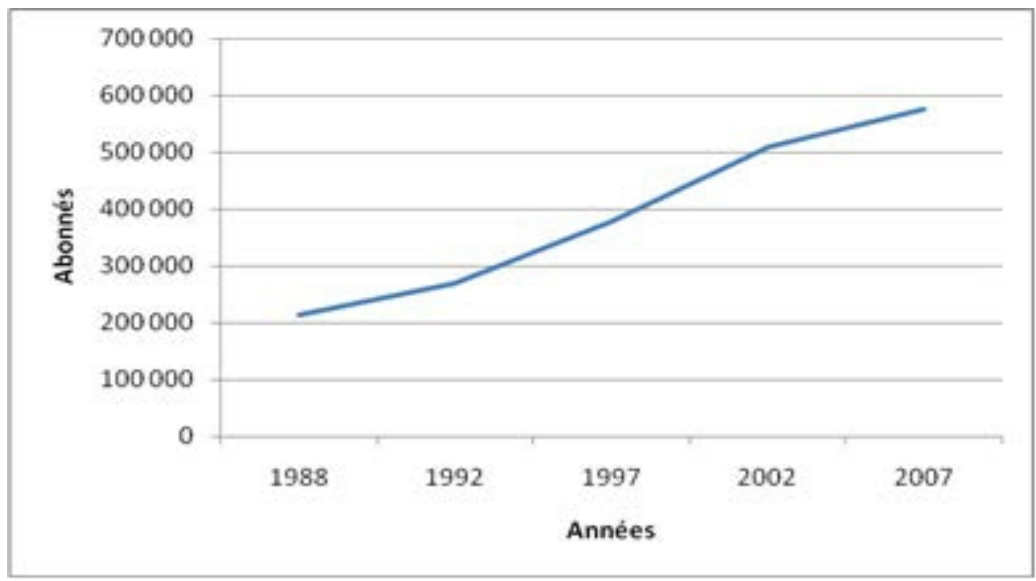

Figure 2 : courbe d’évolution des abonnés de la Sodeci de 1988 à 2007 
Tableau 2 : Données de la SODECI entre 1988 et 2007 au plan national

\begin{tabular}{|c|c|c|c|c|c|}
\hline Années & $\mathbf{1 9 8 8}$ & $\mathbf{1 9 9 2}$ & $\mathbf{1 9 9 7}$ & $\mathbf{2 0 0 2}$ & $\mathbf{2 0 0 7}$ \\
\hline Nombre d'abonnés & 212624 & 267659 & 377750 & 508589 & 576552 \\
\hline Production $\left(\mathrm{km}^{3}\right)$ & 94558 & 102758 & 124241 & 148329 & 170897 \\
\hline Facturation $\left(\mathrm{km}^{3}\right)$ & 80728 & 89824 & 103368 & 120485 & 132759 \\
\hline Consommation unitaire $\left(\mathrm{m}^{3}\right)$ & 387 & 336 & 274 & 237 & 230 \\
\hline
\end{tabular}

Source : SODECI, 2010

\section{1-2-5-Améliorer et préserver le patrimoine (2008-2022)}

La SODECI va signer en octobre 2007, un nouveau contrat d'affermage d'une durée de 15 ans avec l'Etat de Côte d'Ivoire. La convention prévoit que l'Etat délègue une partie de ses responsabilités pour l'exploitation, l'entretien, le renouvellement d'ouvrages existants et la relation avec les usagers à la SODECI. Le financement et la construction de nouveaux ouvrages demeurent la responsabilité de l'Etat. Ainsi, l'Administration a mis en place l'Office National de l'Eau Potable (ONEP) avec pour mission la gestion du patrimoine et le développement des infrastructures. Par ailleurs, le Ministère des Infrastructures Economiques (MIE) a créé la Direction Générale de l'Approvisionnement en Eau (DGAE), chargée notamment de définir la politique de l'alimentation en eau potable et d'assurer la régulation. Les besoins sont considérables et beaucoup de projets d'extension de réseau, de forages ou encore d'adduction qui permettront de renforcer les capacités de production et de distribution d'eau potable restent encore au stade d'études malgré l'urgence de la situation.

\section{2-Les difficultes de la sodeci}

La SODECI, durant son évolution, va connaître deux grandes difficultés qui sont: le surendettement du secteur lorsqu'elle a entrepris d'étendre sa distribution sur le réseau national et la crise militaro-politique de 2002.

\section{2-1-Le surendettement}

En dépit de ces performances opérationnelles et financières, la période (1987-2007) est caractérisée par un surendettement du secteur car les recettes n'arrivaient pas à couvrir les emplois. Malgré un suréquipement en moyens de production et de distribution, le taux de desserte restait faible. Ainsi pour restaurer l'équilibre financier du secteur de l'eau, les autorités prennent des décisions stratégiques pour recentrer les activités de la SODECI essentiellement vers l'hydraulique. Parmi ces mesures, nous avons :

\section{2-1-1-Un nouveau contrat pour l'eau potable}

Les nouveaux investissements seront dorénavant liés aux besoins opérationnels. Le développement des infrastructures devra être axé sur 
l'extension du réseau. Afin de mener à profit la surcapacité existante et de satisfaire la demande croissante engendrée par une population urbaine en pleine explosion, l'Etat va mettre en place un programme de branchements sociaux subventionnés à $100 \%$. En plus, un nouveau Fonds de Développement de l'Eau (FDE) est créé, selon un schéma radicalement différent du fonds précédent. Sa gestion est confiée directement à la SODECI. Le FDE ne fonctionne plus sur emprunts publics, mais au moyen d'une redevance sur le tarif payé par les usagers. Cette nouvelle formule introduit un schéma contractuel hybride entre le contrat d'affermage et la concession. La SODECI est directement responsable des décisions concernant le choix des investissements ainsi que de leur exécution, comme le ferait une concession sans avoir à supporter les risques financiers connexes (SODECI, 2010).

\section{2-1-2-Un nouveau contrat de services pour l'assainissement}

Parallèlement au contrat pour l'eau potable, un contrat de services pour l'assainissement est renégocié par période triennale en 1987. La responsabilité de l'entretien et l'exploitation des réseaux incombent désormais à l'Etat. La SODECI se contentait d'indiquer les travaux nécessaires à réaliser avant exécution. La surtaxe d'assainissement a été supprimée. L'Etat accumulait les dettes et ne payait plus ses factures, ce qui était préjudiciable à la santé financière de la SODECI. En 1999, un compromis a été finalement trouvé, grâce à la signature d'un contrat d'affermage du service d'assainissement de la ville d'Abidjan (remplaçant le contrat de prestation de service) pour une durée de 16 ans. La SODECI prenait alors la totale responsabilité du bon fonctionnement des réseaux et des ouvrages d'assainissement bien que le patrimoine restait la propriété de l'Etat. Son financement était assuré principalement par une redevance applicable aux abonnés du réseau d'eau potable de la ville d'Abidjan. Ce contrat d'affermage et la redevance appliquée amélioraient et favorisaient l'accès au financement des bailleurs de fonds.

\section{2-1-3-Les mesures de rationalisation}

Après le retrait de l'activité de l'hydraulique villageoise, la SODECI a vu ses activités se réduire et a été obligée de rompre le contrat de travail de 250 salariés. En dépit de toutes les difficultés qu'elle a connues, la SODECI a pu maintenir le cap en assurant la desserte en eau potable de 8.8 millions de personnes, de 763 localités (SODECI, 2010). Aussi est-elle devenue la première société de service public certifiée en Afrique avec la norme ISO 9002. 


\section{2-2-Les impacts de la crise militaro-politique de 2002}

Le déclenchement de la guerre en Côte d'Ivoire le 19 septembre 2002 a fortement perturbé les activités opérationnelles et commerciales ; jusqu'à parfois s'interrompre dans plusieurs directions régionales notamment, celles occupées par les forces nouvelles appelées Centre-Nord-Ouest (CNO). Même si un service minimum en matière de production et de dépannage a continué d'y être assuré, la SODECI a très vite installé un comité de crise, chargé de l'accueil des collaborateurs qui ont dû fuir les zones Centre-NordOuest (CNO) et les réintégrer dans les structures à Abidjan, avant de tenter d'assurer la continuité du service. Mais, cette situation sera lourde de conséquences pour l'entreprise qui connaît une baisse de production, du fait des déplacements massifs de populations. Le départ des collaborateurs entraîne l'arrêt des activités commerciales, la facturation ne se fait presque plus, le taux de recouvrement de la SODECI (9\% du chiffre d'affaires global) est en baisse et la fraude en augmentation. Cette chute du chiffre d'affaire est due à l'absence de facturation dans les zones ex-CNO. Le tableau 3 montre la réduction du nombre d'abonnés dans ces zones entre 2002 et 2010.

Tableau 3 : Nombre d'abonnés SODECI dans les ex-zones CNO entre 2002 et 2010

\begin{tabular}{|c|c|}
\hline Années & Nombre d'abonnés \\
\hline 2002 & 85119 \\
\hline 2006 & 82955 \\
\hline 2007 & 70815 \\
\hline 2010 & 72625 \\
\hline
\end{tabular}

Source : SODECI, 2010

La SODECI a subi de nombreux dommages matériels notamment, le pillage de ses locaux d'exploitation, le vol ou la destruction de ses véhicules. Le coût financier sera également important concernant le patrimoine de l'Etat. L’Union Européenne et la Croix Rouge sont venues en aide à l'Etat afin de financer la préservation et la réhabilitation des installations. La dégradation du climat politique aura aussi ses effets néfastes sur l'état d'esprit des travailleurs, avec une forte incidence sur la motivation et la productivité. Cherchant à prévenir les dérives possibles, la SODECI a instauré à cette même époque, un comité de veille sociale. De 2002 à 2004, les facturations et les recouvrements n'ont pas pu être effectués. Plusieurs tentatives et de nombreuses démarches de sensibilisation auprès des autorités politiques et militaires locales ont été entreprises. Une reprise progressive des activités commerciales et techniques a débuté en 2004, mais il a fallu attendre janvier 2009 pour que la facturation reprenne totalement.

Hormis ces difficultés liées aux raisons économiques et politiques, force est de constater que la SODECI n'est pas appréciée par beaucoup d'ivoiriens à cause des pénuries et coupures intempestives d'eau qui ont lieu dans la ville d'Abidjan. Pour eux, la SODECI est une structure incapable de 
fournir de l'eau aux populations et se mue en des escrocs puisque les populations n'ont pas de l'eau dans leur robinet, mais elles reçoivent chaque trois mois, des factures qu'elles sont obligées de payer au risque de voir le compteur enlevé par ses agents. Cette absence généralisée de l'eau potable dans la ville d'Abidjan est aussi source de conflits, comme cela a été le cas à Yopougon-Micao, zone industrielle à l'ouest d'Abidjan. En effet, des bousculades pour s'approvisionner dans des points d'eau de fortune ont dégénéré en affrontements, faisant notamment des blessés parmi les femmes et les enfants. Selon les populations de Yopougon-Micao, elles sont obligées de chercher des points d'eau de fortune et quand elles les trouvent, c'est une ruée et seuls les plus forts arrivent à se faire servir quand les plus faibles sont piétinés et blessés. En dehors des affrontements provoqués par la pénurie d'eau, le manque d'eau potable à Abidjan pose un véritable problème de santé publique. Certains, pour relever un tant soit peu leur niveau de vie, proposent à la consommation des uns et des autres, un peu partout dans la ville, de l'eau en sachet ou des jus en bouteille, conditionnés «on ne sait trop comment ». Une enquête réalisée par les autorités ivoirienne en 2011 a par exemple, révélé que de nombreux sachets d'eau vendus dans la ville proviendraient de puits ou même de la lagune ébrié, d'où les mesures d'interdiction de vente de jus en sachet ou en bouteille et la commercialisation non réglementaire de l'eau en sachet.

Selon le MIE (2012), le calvaire des ménages abidjanais pourrait prendre fin, avec l'existence de nombreux projets visant à leur garantir l'accès continu à l'eau potable. A Angré, quartier de la commune de Cocody, autrefois fortement touché, la construction d'un réservoir de $5000 \mathrm{~m}^{3}$ par le FDE sur fonds propres à hauteur de dix milliards de francs CFA a vu le jour. Cette réalisation a grandement atténué le calvaire des populations auparavant privées d'eau, pendant des périodes s'étalant sur deux ou plusieurs semaines. En attendant, l'usage des puits est déconseillé à cause de la non-existence d'un réseau souterrain d'évacuation des eaux usées partout dans la mégapole et ce, du fait des zones de construction non viabilisées incluant les bidonvilles. En dépit de toutes ses péripéties, la SODECI dessert à ce jour 964 localités et 13745067 habitants soit un taux de couverture de 68\% (ONEP, 2015).

Cette sévère crise politique qui a frappé le pays a fait perdre la place de référence qu'occupait la SODECI en Afrique en matière de gestion des services publics d'eau et d'assainissement. Pour atteindre cet objectif, elle va mettre en place une politique stratégique qui va couvrir la période 2015/2019. 


\section{Conclusion}

La SODECI est née en terre d'Eburnie dans le but de favoriser un accès à l'eau potable aux populations. Elle va connaître un développement remarquable à travers l'application des priorités qu'elle s'est fixée. Malheureusement, dans son évolution, elle va être confrontée à deux crises majeures qui vont la fragiliser. Malgré cela, elle a pu faire face aux différents challenges en desservant 964 localités et 13745067 habitants soit un taux de couverture de $68 \%$. De ce fait, elle apparaît comme un outil stratégique dans la distribution de l'eau en Côte d'Ivoire. Pour se repositionner en tant que leader africain en matière de gestion des services d'eau et d'assainissement, elle a mis en œuvre des mécanismes performants répondant aux normes internationales. Ainsi, elle a adopté plusieurs stratégies qui se résument en six points essentiels : demeurer le meilleur partenaire de l'Etat en matière de gestion des services publics de l'eau et de l'assainissement; renforcer et rendre plus visible son image d'entreprise citoyenne engagée dans le développement durable ; améliorer les relations avec les clients et les autres parties prenantes ; innover dans tous les domaines (technique, commercial, social ...) et renforcer le bien-être de ses collaborateurs.

\section{References:}

DIABAGATE A., (2012), Importance numérique et répartition spatiale des ethnies et nationalités dans l'agglomération d'Abidjan, Thèse unique de Doctorat en géographie, Université Félix Houphouët Boigny- Abidjan, IGT, $435 \mathrm{p}$.

KOUKOUGNON W., (2012) : Milieu urbain et accès à l'eau potable : cas de Daloa (Centre-Ouest de la Côte d'Ivoire), Thèse unique de Doctorat en géographie, Université Félix Houphouët Boigny- Abidjan, IGT, 370 p.

MINISTERE DES INFRASTRUCTURES ECONOMIQUES, (2012) : tout savoir sur les problèmes de l'eau potable en Côte d'Ivoire, MIE, Abidjan, 9p. ONEP, (2015), Rapport de suivi du patrimoine, ONEP, Abidjan, 15p.

ONU, (2015), Objectifs du millénaire pour le développement, rapport 2015, 78p.

SODECI, (2010), Cinquante ans au service public de la Côte d'Ivoire, Document spécial, Abidjan, 51p. 